OPEN ACCESS

Edited by:

Enrique Cadenas,

University of Southern California,

United States

Reviewed by:

Ian M. McDonough,

University of Alabama, United States

Ramesh Kandimalla,

Texas Tech University Health Sciences

Center, United States

*Correspondence:

Sheryl L. Coley

slcoley28@gmail.com

Received: 01 March 2019

Accepted: 13 May 2019

Published: 31 May 2019

Citation:

Green-Harris G, Coley SL, Koscik RL, Norris NC, Houston SL, Sager MA, Johnson SC and Edwards DF (2019) Addressing Disparities in Alzheimer's

Disease and African-American

Participation in Research: An

Asset-Based Community

Development Approach

Front. Aging Neurosci. 11:125

doi: 10.3389/fnagi.2019.00125

\section{Addressing Disparities in Alzheimer's Disease and African-American Participation in Research: An Asset-Based Community Development Approach}

\author{
Gina Green-Harris ${ }^{1,2}$, Sheryl L. Coley ${ }^{3 *}$, Rebecca L. Koscik ${ }^{3}$, Nia C. Norris ${ }^{1}$, \\ Stephanie L. Houston ${ }^{1}$, Mark A. Sager ${ }^{3}$, Sterling C. Johnson ${ }^{3,4,5}$ and \\ Dorothy Farrar Edwards 5,6,7
}

${ }^{1}$ Wisconsin Alzheimer's Institute Regional Milwaukee Office, University of Wisconsin School of Medicine and Public Health, Milwaukee, WI, United States, ${ }^{2}$ Center for Community Engagement and Health Partherships, University of Wisconsin School of Medicine and Public Health, Madison, WI, United States, ${ }^{3}$ Wisconsin Alzheimer's Institute, University of Wisconsin School of Medicine and Public Health, Madison, WI, United States, ${ }^{4}$ Geriatric Research Education and Clinical Center, Wm. S. Middleton Veterans Hospital, Madison, WI, United States, ${ }^{5}$ Wisconsin Alzheimer's Disease Research Center, University of Wisconsin School of Medicine and Public Health, Madison, WI, United States, ${ }^{6}$ Department of Kinesiology, School of Education, University of Wisconsin, Madison, WI, United States, ${ }^{7}$ Department of Medicine, University of Wisconsin School of Medicine and Public Health, Madison, WI, United States

Keywords: African Americans, Alzheimer's disease, health disparities, recruitment, community engagement

\section{INTRODUCTION}

Alzheimer's Disease and related dementias (ADRD) remain a worldwide burden to address for persons with ADRD, their family members, and others at risk. ADRD should be recognized as a public health priority (Barnes, 2019), particularly among African Americans for several reasons. Currently, African Americans have 2-4 times the risk of developing ADRD than non-Hispanic Whites (Barnes and Bennett, 2014; McDonough, 2017). Given the higher presence of co-morbid conditions and associated health disparities when comparing African Americans to other racial and ethnic groups (Dillworth-Anderson and Boswell, 2007; Hill et al., 2015), African Americans can have worse physical health and quality-of-life. These factors can also increase costs of care for persons with ADRD and their family members. African Americans could be further burdened by significantly higher costs of care than Whites (Gilligan et al., 2012) and lack of adequate insurance.

Despite these disparities, African Americans are still underrepresented in ADRD research. Cultural and institutional barriers include primary reliance on passive recruitment strategies (e.g., postings, flyers, database recruitment) not tailored to communities of color, implicit bias, and mistrust of medical communities, academic institutions, and researchers (Ballard et al., 2010; Scharff et al., 2010; Williams et al., 2010; Shin and Doraiswamy, 2016). Investigator inclusion and participation criteria deemed more feasible for White participants (ex. Medical eligibility criteria, job restrictions, time, and financial requirements to go to study sites) also undermine or limit the participation of African Americans and other participants of color (Wendler et al., 2006; Konkel, 2015).

Using asset-based community development (ABCD) approaches (Kretzmann and McKnight, 1993) can lead to improved access and delivery of care and ADRD knowledge among African 
Americans, increased social support for African-American caregivers and elders living with or at risk for ADRD, and increased African-American representation in neuroscience research. In this article, we describe the development and implementation of an ABCD approach for increasing AfricanAmerican representation in the Wisconsin Registry for Alzheimer's Project (WRAP).

\section{WRAP AND INITIAL RECRUITMENT}

WRAP started in November 2001 with participants initially recruited from adult children of parents with ADRD receiving care from Wisconsin Alzheimer's Institute (WAI) memory diagnostic clinics (Sager et al., 2005). WRAP remains one of the largest and longest-running longitudinal observational cohort studies for adult children of persons with Alzheimer's Disease $(N>1,100$ with parental history of $\mathrm{AD}$ and $>400$ persons without known parental history) for identifying early declines in cognition and examining midlife factors and biomarkers related to the disease (Johnson et al., 2018). Initial recruitment strategies included educational presentations, clinic referrals, newspaper advertisements, and emails sent to University of Wisconsin-Madison (UW) alumni. Using these recruitment methods reached few African Americans, particularly alumni recruitment given the historically low rates of alumni of color at predominantly White universities including UW. Only six African Americans (0.8\% of 711) enrolled in WRAP by 2005.

To address this recruitment disparity, strategies of recruiting from communities that are more diverse and hiring a more diverse study team were implemented. Specifically, Milwaukee became a recruitment site in 2006; African Americans and other residents of color constituted over $50 \%$ of Milwaukee's population (United States Census Bureau, 2006). A Milwaukeebased office was established and African-American program and outreach managers were hired to implement recruitment strategies in Milwaukee that were similar to those used in Madison (e.g., clinic-based recruitment and advertisements). African-American recruitment at the end of 2007 remained low ( $<2 \%$ of WRAP) despite implementing these strategieschanging recruitment strategies was identified as an important next step.

\section{WAI-MILWAUKEE ASSET-BASED COMMUNITY DEVELOPMENT MODEL}

In 2008, new WAI-Milwaukee leadership who had lifelong connections to Milwaukee developed and implemented an ABCD approach to African-American recruitment. This approach patterns Kretzmann and McKnight (1993) ABCD community-engagement principles through connecting residents with culturally-tailored programs and services with community-identified needs. ABCD maintains that initiatives from exterior sources such as university-based research projects will be most effective when community assets are leveraged at full capacity. Sustainable community development stems from (1) assessing resources, skills, and experience available in community members, (2) organizing communities around issues that move members into action, and (3) community members determining priorities and taking appropriate action. This community development leads to increased services provided to communities and increased representation of African Americans in research. Table 1 (Panel A) illustrates the core aspects of WAI-Milwaukee's ABCD approach.

Several strategies were initiated through this approach (Table 1, Panel B), with the identification of community assets and key stakeholders as the first step. Through grassroot networking efforts, the new leadership team identified key stakeholders among African-American residents affected by ADRD. This networking led to the identification of $10-15$ African-American residents and caregivers with diverse roles in their respective communities. Stakeholders included an AfricanAmerican physician, public health officials, and local media professionals who cared for family members with ADRD. The WAI-Milwaukee leadership team and stakeholders developed a community advisory board (CAB) in 2008 that counseled the WAI-Milwaukee team on culturally-tailored outreach and engagement, recruitment strategies, and potential resources that could be utilized for community-based efforts. Developing CABs is an effective strategy for overcoming barriers related to power differentials between researchers and communities in which the research is being conducted (Dancy et al., 2004).

Next, the Milwaukee Health Services Memory Diagnostic Clinic was developed with local infrastructure funding through the university's Wisconsin Partnership Program. Led by an African-American physician, it became the first memory diagnostic clinic in the nation located in a federallyqualified medical center. Memory clinic patients received complementary health education, dementia classes, and lifestyle intervention courses. Working with clinics within the WAI clinic network gave WAI-Milwaukee credibility in AfricanAmerican communities for being a trusted resource of clear and relevant information.

This credibility provided a platform for WAI-Milwaukee to become part of the community social fabric (Jeste et al., 2016) to deliver patient-centered training and community resources to engage African-American elders. For example, $\mathrm{CAB}$ meetings revealed residents' desire for advocacy training for themselves and their family members to use during provider interactions. Provider education was also identified as a critical need given community members' reports of insufficient education received from providers about health conditions. Based on community feedback, primary care and allied health workers received information from WAI-Milwaukee about articulating health information back to patients with inclusion of family members and considerations for enhancing African-American patients' trust in the medical system.

For developing culturally-tailored programming, the WAIMilwaukee team identified and applied community-based culturally-tailored aspects into educational initiatives originally created for mainstream audiences. Incorporating communities' value systems and historical perspectives means identifying the norms, continuously asking questions, and becoming educated by community members in a cyclical process. As a leadership 
TABLE 1 | WAI-Milwaukee Asset-Based Community Development (ABCD) Approach, examples of community activities and current outcomes.

\begin{tabular}{|c|c|c|}
\hline Panel A: Core Aspects of ABCD Approach & Panel B: Examples of Activities & Panel C: Outcomes \\
\hline $\begin{array}{l}\text { Community assessment } \\
\text { - Identify the community and their assets } \\
\text { - Identify key community stakeholders } \\
\text { - Address the community needs and wants }\end{array}$ & $\begin{array}{l}\text { Outreach \& Education } \\
\text { - Breaking the Silence Annual Breakfast and } \\
\text { Community Workshops } \\
\text { - The "Amazing Grace" Chorus Family Support } \\
\text { Program } \\
\text { - Annual Faith-Based ADRD Initiatives }\end{array}$ & $\begin{array}{l}\text { Stigma Reduction } \\
\text { Increased community awareness of ADRD }\end{array}$ \\
\hline $\begin{array}{l}\text { Community engagement (WAI-Milwaukee becoming } \\
\text { part of community "social fabric") } \\
\text { - Invest time in the community } \\
\text { - Provide resources identified by the community } \\
\text { - Address barriers through service }\end{array}$ & $\begin{array}{l}\text { Coordination of Medical \& Social Services } \\
\text { Milwaukee Health Services Center \& Clinic } \\
\text { Network Development } \\
\text { Culturally inclusive professional training }\end{array}$ & $\begin{array}{l}\text { Increased access of community members to } \\
\text { comprehensive care and support services }\end{array}$ \\
\hline $\begin{array}{l}\text { Community involvement } \\
\text { - Recognize community members as experts } \\
\text { - Validate community members' perspectives } \\
\text { - Build relationships between community members, } \\
\text { researchers, and health professionals } \\
\text { - Community members provide counsel to } \\
\text { WAl-Milwaukee and researchers }\end{array}$ & $\begin{array}{l}\text { Community Advisory Board development and } \\
\text { sustenance }\end{array}$ & $\begin{array}{l}\text { Academic-community partnership in initiatives } \\
\text { Increased community awareness of importance of } \\
\text { research } \\
\text { Increased African-American participation in research }\end{array}$ \\
\hline
\end{tabular}

team member, an African-American family care coordinator talked with families in their homes to identify barriers and connect families to services and programs while incorporating cultural respect (ex. Addressing titles, meeting them during times and events convenient for them). Through preliminary education sessions, community members expressed their readiness to talk about brain health-oriented solutions before discussing ADRD. Milwaukee CAB members facilitated these education sessions that enhanced the co-learning process between AfricanAmerican residents and WAI-Milwaukee. With the support of reputable African-American organizations, these education sessions led to larger health promotion efforts in various residential areas of the city, including lower-income highrise apartments. To address social support desires expressed by caregivers, programming for African-American caregivers and family members with dementia included the development of the Amazing Grace Chorus Family Support Program. The leadership team developed this program with local music teachers through adapting current mainstream music therapy interventions (Mittelman and Papayannopoulou, 2018) designed to improve quality of life and social support for the caregivers and family members with ADRD (Refer to Table 1, Panel B for more programming examples).

\section{WRAP RECRUITMENT LESSONS FOR SUCCESS}

This reciprocal community-university partnership yielded favorable outcomes to date (Table 1, Panel C). Through this partnership, WAI-Milwaukee gathered valuable information on community priorities from African-American members for informing community-engagement activities, providing beneficial services for community members, and reducing stigma about ADRD. Subsequently, WAI-Milwaukee built trust among community members and providers and commitment to research. From 2008 to 2012, African-American participation in WRAP increased more than $400 \%$ from 0.8 to $~ 8.0 \%$ of the study sample (131 of 1,573). Recruitment of new African-American participants plateaued for several years when budget constraints allowed primarily for return visits of existing participants; recent funding allows resumed recruitment of more African Americans.

Several lessons are worth consideration for increasing African-American research participation. Although similar strategies have been used for aging health studies [ex. (Gluck et al., 2018)], our approach emphasizes meeting the community members' needs first through service before discussing participation in research. Through an iterative process with community members, the leadership team addressed other issues related to ADRD before explicitly addressing ADRD. Recruiting African-Americans to participate in research was the last step; notably, families agreed to participate in WRAP as reciprocity for the services received from WAI-Milwaukee. This approach continues as an iterative process to meet the needs of the communities and to retain members' interest and participation in research.

Investment in relationship building remains essential for recruitment (Barnes, 2019), and multiple stakeholders must have shared vision and mutual trust. $\mathrm{CAB}$ community members shared recruitment responsibilities with the WAI-Milwaukee team at community centers and events, and the WAI-Milwaukee team shared recruitment responsibilities with WAI clinic network physicians in the clinics. African-American recruitment was primarily word-of-mouth with the shared message that WRAP participation helps African Americans receive better clinical diagnoses, therefore African Americans would benefit from research participation. WAI-Milwaukee credits the communities for building the model for WRAP recruitment.

Next, alternate funding streams apart from government funding are feasible resources for community engagement infrastructure building and related activities that enhance research efforts. WAI-Milwaukee credits philanthropic efforts 
(Bader Philanthropies, Wisconsin Partnership Program) which allowed room for capacity-building, community engagement, and developing relationships outside of rigid timeframes of traditional research funding. Building the strategic framework and developing capacity is critical before focusing on obtaining research funding through traditional means.

Finally, sustainability must be considered before beginning any community-engaged research initiative. Community and philanthropic relationships facilitate the sustainability of WAIMilwaukee's programming. Philanthropic funding draws other types of funding agencies to contribute to WAI-Milwaukee's infrastructure. Through leveraging philanthropic support in garnering other funding, infrastructure is sustained for retaining ongoing relationships and forging new relationships with community entities.

\section{CONCLUSION}

WAI-Milwaukee applied ABCD principles to improve recruitment of African Americans into longitudinal research for $\mathrm{ADRD}$. Initiatives grounded in $\mathrm{ABCD}$ approaches can potentially improve quality of care, reduce costs, decrease burden, improve quality-of-life and subsequently increase interest in research participation among African Americans. $\mathrm{ABCD}$ approaches can also potentially increase interest in therapeutic interventions among African-Americans that reduce the burden of $\mathrm{ADRD}$, as exemplified by the family care coordination and development of the Amazing Grace Chorus Family Support Program. Finally, future models grounded in

\section{REFERENCES}

Ballard, E. L., Gwyther, L. P., and Edmonds, H. L. (2010). Challenges and opportunities: recruitment and retention of African Americans for Alzheimer disease research: lessons learned. Alzheimer Dis. Assoc. Disord. 24, S19-S23. doi: 10.1097/WAD.0b013e3181f12432

Barnes, L. L. (2019). Biomarkers for Alzheimer dementia in diverse racial and ethnic minorities: a public health priority. JAMA Neurol. 76, 251-253. doi: 10.1001/jamaneurol.2018.3444

Barnes, L. L., and Bennett, D. A. (2014). Alzheimer's disease in African Americans: risk factors \& challenges for the future. Health Aff. 33, 580-586. doi: $10.1377 /$ hlthaff.2013.1353

Dancy, B. L., Wilbur, J., Talashek, M., Bonner, G., and Barnes-Boyd, C. (2004). Community-based research: barriers to recruitment of African Americans. Nurs. Outlook 52, 234-240. doi: 10.1016/j.outlook.2004.04.012

Dillworth-Anderson, P., and Boswell, G. (2007). "Cultural diversity and aging: ethnicity, minorities, and subcultures," in The Blackwell Encyclopedia of Sociology, Vol. II, ed G. Ritzer (Oxford: Blackwell Publishing), 898-902. doi: 10.1002/9781405165518.wbeosc173

Gilligan, A. M., Malone, D. C., Warholak, T. L., and Armstrong, E. P. (2012). Health disparities in cost of care in patients with Alzheimer's disease: an analysis across 4 state Medicaid populations. Am. J. Alzheimers Dis. Other Dement. 28, 84-92. doi: 10.1177/1533317512467679

Gluck, M. A., Shaw, A., and Hill, D. (2018). Recruiting older African Americans to brain health and aging research through community engagement. Generations $42,78-82$.

Hill, C. V., Pérez-Stable, E. J., Anderson, N. A., and Bernard, M. A. (2015). The National Institute on aging health disparities research framework. Ethn. Dis. 25, 245-254. doi: 10.18865/ed.25.3.245

Jeste, D. V., Blazer, D. G. II., Buckwalter, K. C., Cassidy, K. L. K., Fishman, L., Gwyther, L. P., et al. (2016). Age-friendly communities initiative: public
$\mathrm{ABCD}$ approaches can facilitate African-American recruitment into ADRD studies for the improvement of the field and decreasing disparities.

\section{AUTHOR CONTRIBUTIONS}

GG-H, SC, and RK drafted this manuscript and conducted the literature research. GG-H, SH, NN, and DE conceived the vision and implemented the asset-based community development approach for WRAP African-American recruitment for which this article is based. SH, NN, MS, SJ, and DE reviewed this article and provided content revisions. All authors provided their final agreement for submission of this manuscript.

\section{FUNDING}

Funding came from Bader Philanthropies, University of Wisconsin School of Medicine and Public Health Wisconsin Partnership Program (WPP2782), National Institutes of Health (R01 AG027161 \& P50 AG033514), University of Wisconsin Institute for Clinical and Translational Research (1UL1TR002373), Wisconsin Geriatric Education Center, and internal Wisconsin Alzheimer's Institute resources.

\section{ACKNOWLEDGMENTS}

The authors gratefully acknowledge Milwaukee Health Services Inc., WAI-Milwaukee Community Advisory Board, and WRAP participants for their continued contributions in this research.

health approach to promoting successful aging. Am. J. Geriatr. Psychiatry 24, 1158-1170. doi: 10.1016/j.jagp.2016.07.021

Johnson, S. C., Koscik, R. L., Jonaitis, E. M., Clark, L. R., Mueller, K. D., Berman, S. E., et al. (2018). The Wisconsin Registry for Alzheimer's Prevention: a review of findings and current directions. Alzheimers Dement. 10, 130-142. doi: 10.1016/j.dadm.2017.11.007

Konkel, L. (2015). Racial and ethnic disparities in research studies: the challenge of creating more diverse cohorts. Environ. Health Perspect. 123, A297-A302. doi: 10.1289/ehp.123-A297

Kretzmann, J. P., and McKnight, J. (1993). Building Communities From the Inside Out: A Path Toward Finding and Mobilizing a Community's Assets. Evanston, IL: The Asset-Based Community Development Institute, School of Education and Social Policy, Northwestern University.

McDonough, I. M. (2017). Beta-amyloid and cortical thickness reveal racial disparities in preclinical Alzheimer's disease. Neuroimage Clin. 16, 659-667. doi: 10.1016/j.nicl.2017.09.014

Mittelman, M. S., and Papayannopoulou, P. M. (2018). The Unforgettables: a chorus for people with dementia with their family members and friends. Int. Psychogeriatr. 30, 779-789. doi: 10.1017/S1041610217001867

Sager, M. A., Hermann, B., and La Rue, A. (2005). Middle-aged children of persons with Alzheimer's disease: APOE genotypes and cognitive function in the Wisconsin Registry for Alzheimer's Prevention. J. Geriatr. Psychiatry Neurol. 18, 245-249. doi: 10.1177/0891988705 281882

Scharff, D. P., Mathews, K. J., Jackson, P., Hoffsuemmer, J., Martin, E., and Edwards, D. (2010). More than Tuskegee: understanding mistrust about research participation. J. Health Care Poor Underserv. 21, 879-897. doi: 10.1353/hpu.0.0323

Shin, J., and Doraiswamy, P. M. (2016). Underrepresentation of AfricanAmericans in Alzheimer's trials: a call for affirmative action. Front. Aging Neurosci. 8:123. doi: 10.3389/fnagi.2016.00123 
United States Census Bureau (2006). 2006 American Community Survey: ACS Demographic and Housing Estimates. Milwaukee, WI. Available online at: https://factfinder.census.gov (retrieved April 12, 2018).

Wendler, D., Kington, R., Madans, J., Van Wye, G., Christ-Schmidt, H., Pratt, L. A., et al. (2006). Are racial and ethnic minorities less willing to participate in health research? PLoS Med. 3:e19. doi: 10.1371/journal.pmed. 0030019

Williams, M. W., Scharff, D. P., Mathews, K. J., Hoffsuemmer, J. S., Jackson, P., Morris, J. C., et al. (2010). Barriers and facilitators of African American participation in Alzheimer's disease biomarker research. Alzheimer Dis. Assoc. Disord. 24, S24-S29. doi: 10.1097/WAD.0b013e3181f14a14
Conflict of Interest Statement: The authors declare that the research was conducted in the absence of any commercial or financial relationships that could be construed as a potential conflict of interest.

Copyright (c) 2019 Green-Harris, Coley, Koscik, Norris, Houston, Sager, Johnson and Edwards. This is an open-access article distributed under the terms of the Creative Commons Attribution License (CC BY). The use, distribution or reproduction in other forums is permitted, provided the original author(s) and the copyright owner(s) are credited and that the original publication in this journal is cited, in accordance with accepted academic practice. No use, distribution or reproduction is permitted which does not comply with these terms. 